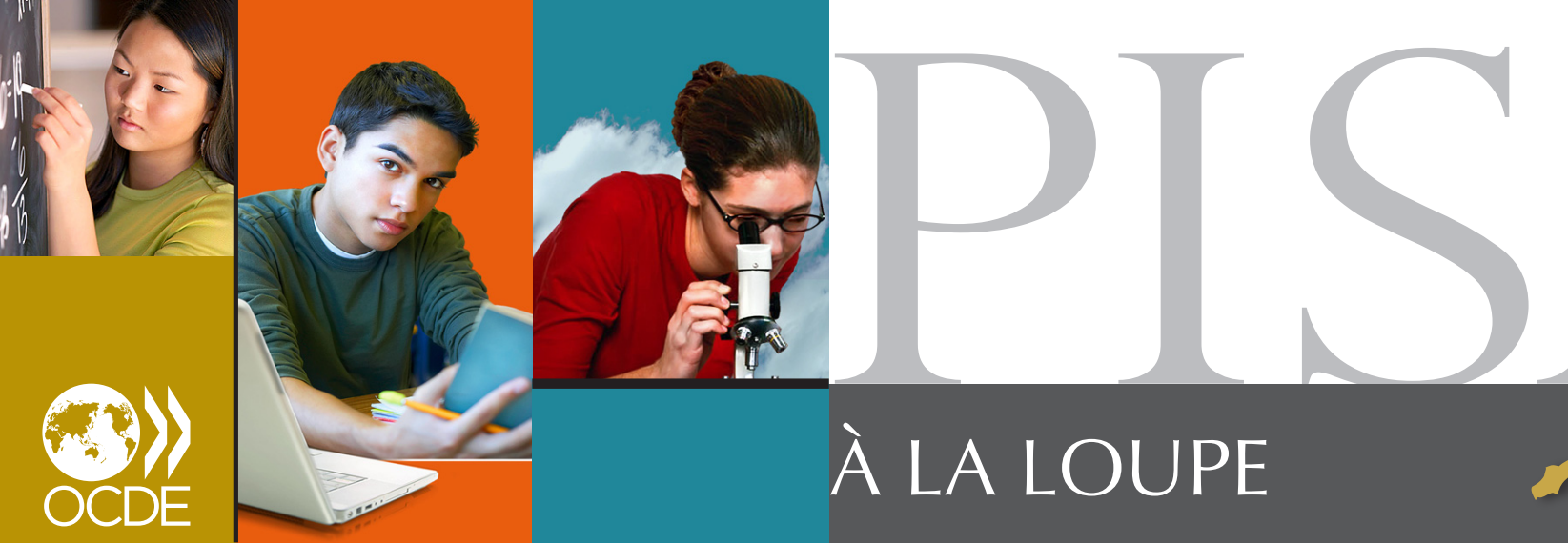

politiques d'éducation politiques d'éducation politiques d'éducation politiques d'éducation politiques d'éducation politiques d'éducation politiques d'éducation

\title{
Garçons et filles sont-ils également prêts à affronter l'ère numérique?
}

- Plus de $17 \%$ des élèves se classent parmi les plus performants en compréhension de l'écrit électronique en Australie, en Corée et en Nouvelle-Zélande, contre moins de 3 \% en Autriche, au Chili et en Pologne.

- En moyenne, les filles sont plus performantes que les garçons en compréhension de l'écrit électronique; toutefois, l'écart entre les sexes est moindre qu'en compréhension de l'écrit sur papier.

- Parmi les garçons et les filles dont le niveau de performance en compréhension de l'écrit sur papier est similaire, les garçons ont, en général, de meilleures compétences en navigation sur support électronique et obtiennent donc de meilleurs résultats en compréhension de l'écrit électronique.

Les technologies de l'information et de la communication révolutionnent non seulement la vitesse de transmission des informations, mais aussi les modalités de diffusion et de réception de ces dernières. Les innovations technologiques modifient en profondeur les types de compétences requises à l'heure actuelle sur le marché du travail et les catégories d'emplois offrant le meilleur potentiel de croissance. La plupart de ces emplois nécessitent désormais une certaine familiarité avec la navigation sur support électronique, voire la maîtrise de cette dernière qui demande aux lecteurs de déterminer eux-mêmes la structure de ce qu'ils lisent au lieu de suivre simplement l'ordre pré-établi du texte, comme dans un livre.

En règle générale, les élèves qui sont de bons L'enquête PISA 2009 a évalué non seulement la capacité lecteurs sur papier le sont aussi sur écran. des élèves de 15 ans à extraire et analyser des informations qu'ils trouvent en lisant des textes sur papier, mais aussi leurs compétences en compréhension de l'écrit électronique. L'enquête PISA montre que certains pays ont réussi bien mieux que d'autres à doter leurs élèves des compétences nécessaires pour participer pleinement à l'ère numérique. Ainsi, plus de $17 \%$ des élèves se classent parmi les plus performants en compréhension de l'écrit électronique en Australie, en Corée et en Nouvelle-Zélande, contre moins de 3 \% en Autriche, au Chili et en Pologne. La Corée a récemment lancé le programme «Smart Education » qui prévoit la numérisation de l'ensemble des manuels scolaires et des évaluations à l'horizon 2015, la construction d'infrastructures scolaires ou l'amélioration de ces dernières afin de les équiper des nouvelles technologies, et la formation des enseignants à l'utilisation de ces technologies.

Bien que, en moyenne, la performance des élèves en compréhension de l'écrit électronique soit étroitement liée à la performance en compréhension de l'écrit sur papier, dans certains pays, tels que l'Australie et la Corée, les élèves sont nettement plus performants en compréhension de l'écrit électronique qu'en compréhension de l'écrit sur papier, tandis que dans d'autres pays, notamment en Hongrie, en Pologne et, dans les pays partenaires, en Colombie, on observe la situation inverse. 
Pourcentage de garçons et de filles parmi les élèves les moins performants en compréhension de l'écrit électronique et de l'écrit sur papier

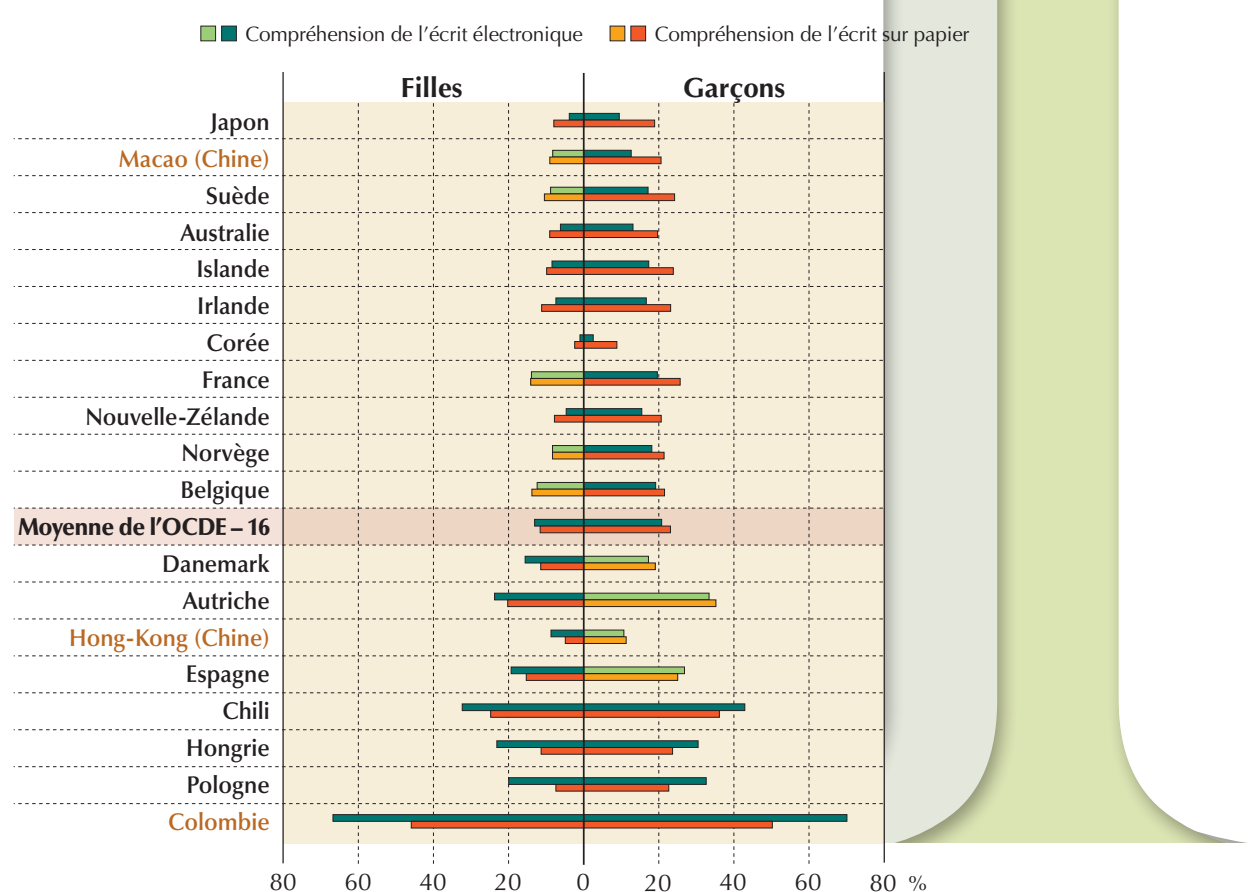

Remarque : les écarts en points de pourcentage (entre la proportion de filles/garçons parmi les élèves les moins performants en compréhension de l'écrit électronique et la proportion de filles/garçons parmi les élèves les moins performants en compréhension de l'écrit sur papier) qui ne sont pas statistiquement significatifs sont indiqués en couleur plus claire.

Les pays sont classés par ordre croissant de l'écart en points de pourcentage entre la proportion de garçons parmi les élèves les moins performants en compréhension de l'écrit électronique et la proportion de garçons parmi les élèves les moins performants en compréhension de l'écrit sur papier.

Source : Base de données PISA 2009 de l'OCDE.

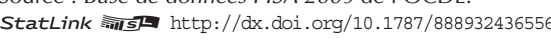

\section{Mais l'écart entre les sexes est moindre qu'en compréhension de l'écrit sur papier..}

L'évaluation a révélé certaines différences intéressantes entre les filles et les garçons en termes de compétences sur support électronique. Si les filles sont plus performantes que les garçons en compréhension de l'écrit sur papier et de l'écrit électronique, l'écart entre les sexes est en général moins prononcé en compréhension de l'écrit électronique. En moyenne, dans les 16 pays de l'OCDE qui ont pris part aux deux évaluations, les filles devancent les garçons de 38 points - soit l'équivalent d'une année d'études - en compréhension de l'écrit sur papier, mais de 24 points en compréhension de l'écrit électronique.

Cette différence s'observe plus nettement aux deux extrémités de l'échelle de compétence, soit parmi les élèves les moins performants et les élèves les plus performants. Ainsi, au Chili, en Hongrie, en Pologne et, dans les pays partenaires, en Colombie, le pourcentage de filles se classant parmi les élèves les moins performants est plus élevé en compréhension de l'écrit électronique qu'en compréhension de l'écrit sur papier. Toutefois, en Australie, en Corée, en Irlande, en Islande, au Japon et en Nouvelle-Zélande, le pourcentage de filles se classant parmi les élèves les moins performants est moins élevé en compréhension de l'écrit

Les élèves les moins performants en compréhension de l'écrit sur papier sont ceux qui n'ont pas atteint le niveau seuil de performance en compréhension de l'écrit. Ils sont capables d'identifier l'idée principale d'un texte portant sur un thème familier et d'établir un lien entre ces informations et leurs connaissances courantes.

Les élèves les moins performants en compréhension de l'écrit électronique sont capables de localiser et d'interpréter des informations bien définies, souvent en rapport avec des contextes familiers. Ils peuvent naviguer entre un nombre limité de sites s'ils ont reçu des instructions explicites.

électronique qu'en compréhension de l'écrit sur papier. L'inverse s'observe chez les garçons. En Australie, en Belgique, en Corée, en France, en Irlande, en Islande, au Japon, en Suède et, dans les économies partenaires, 
à Macao (Chine), le pourcentage de garçons se classant parmi les élèves les moins performants est nettement moins élevé en compréhension de l'écrit électronique qu'en compréhension de l'écrit sur papier.

Les élèves les plus performants en compréhension de l'écrit sur papier sont capables de gérer des idées qui ne leur sont pas familières, en présence d'informations concurrentes saillantes, et de produire des catégories abstraites à des fins d'interprétation.

Les élèves les plus performants en compréhension de l'écrit électronique sont capables de localiser, analyser et évaluer de façon critique des informations dont le contexte ne leur est pas familier en présence d'ambiguïtés. Ils peuvent aussi naviguer entre plusieurs sites sans y être explicitement invités et traiter des textes se présentant sous différents formats.
La plus faible proportion de filles parmi les élèves les plus performants en compréhension de l'écrit électronique par rapport à la compréhension de l'écrit sur papier contribue également à la réduction de l'écart entre les sexes en compréhension de l'écrit électronique. Cette différence est particulièrement marquée en Autriche, en France, au Japon, en Norvège, en Pologne et, dans les économies partenaires, à Hong-Kong (Chine). Par contraste, en Australie, en Corée et en Nouvelle-Zélande, le pourcentage de filles parmi les élèves les plus performants est plus élevé en compréhension de l'écrit électronique qu'en compréhension de l'écrit sur papier.

\section{indice possible d'une plus grande aisance des garçons sur support électronique.}

Quel que soit le pays, l'accroissement du pourcentage d'élèves les plus performants en compréhension de l'écrit électronique par rapport à la compréhension de l'écrit sur papier est toujours plus important parmi les garçons que parmi les filles ; il en va de même pour la réduction du pourcentage d'élèves les moins performants.

\section{Pourcentage de garçons et de filles parmi les élèves les plus performants en compréhension de l'écrit électronique et de l'écrit sur papier}

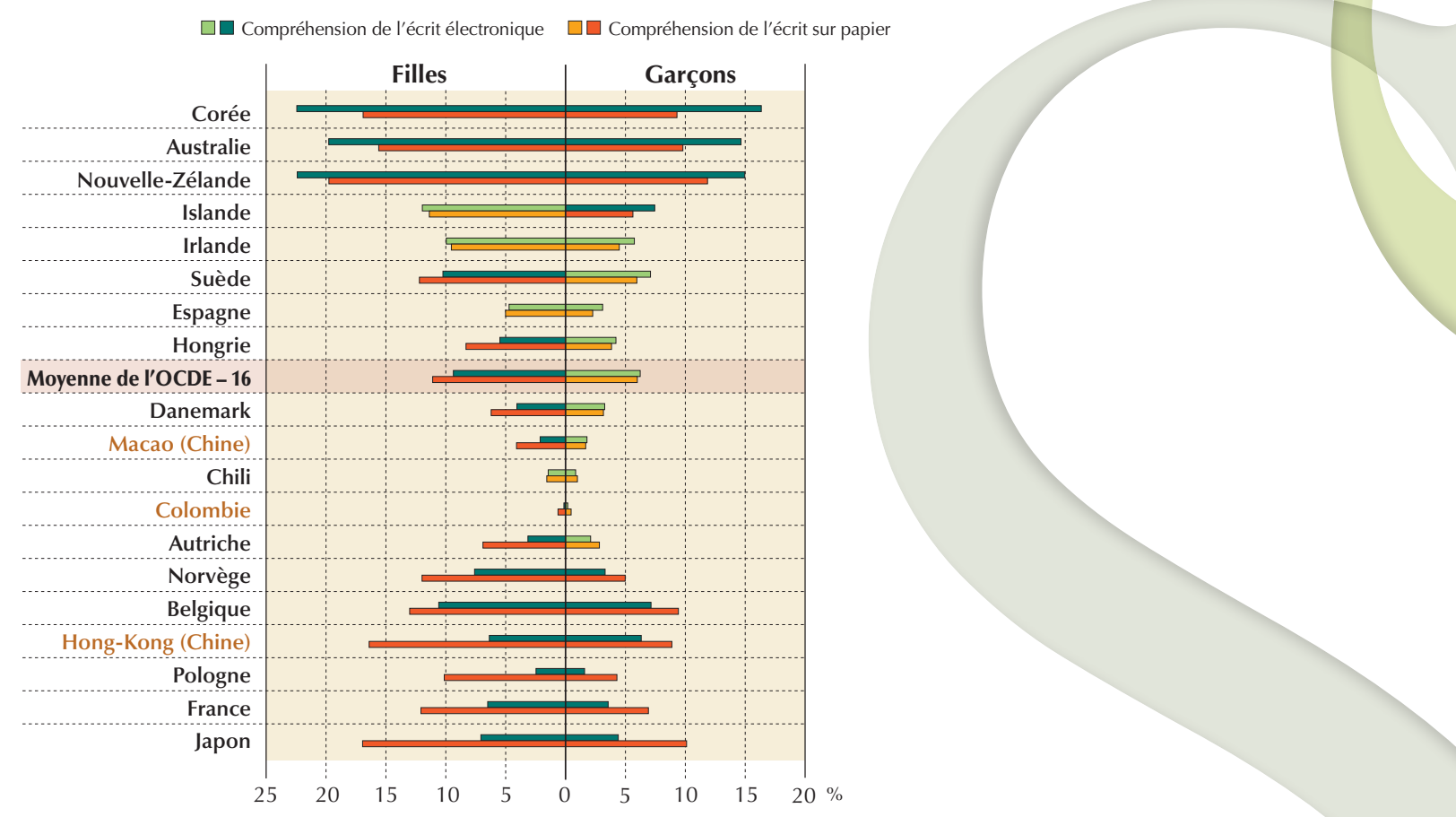

Remarque : les écarts en points de pourcentage (entre la proportion de filles/garçons parmi les élèves les plus performants en compréhension de l'écrit électronique et la proportion de filles/garçons parmi les élèves les plus performants en compréhension de l'écrit sur papier) qui ne sont pas statistiquement significatifs sont indiqués en couleur plus claire.

Les pays sont classés par ordre décroissant de l'écart en points de pourcentage entre la proportion de garçons parmi les élèves les plus performants en compréhension de l'écrit électronique et la proportion de garçons parmi les élèves les plus performants en compréhension de l'écrit sur papier.

Source : Base de données PISA 2009 de l'OCDE.

StatLink 䨛 1 http: //dx.doi.org/10.1787/888932436556 


\section{Ade $P$}

\section{Comparaison de l'écart entre les sexes (en faveur des filles) en compréhension de l'écrit électronique et de l'écrit sur papier}

$\square$ Compréhension de l'écrit électronique $\quad \square \square$ Compréhension de l'écrit sur papier

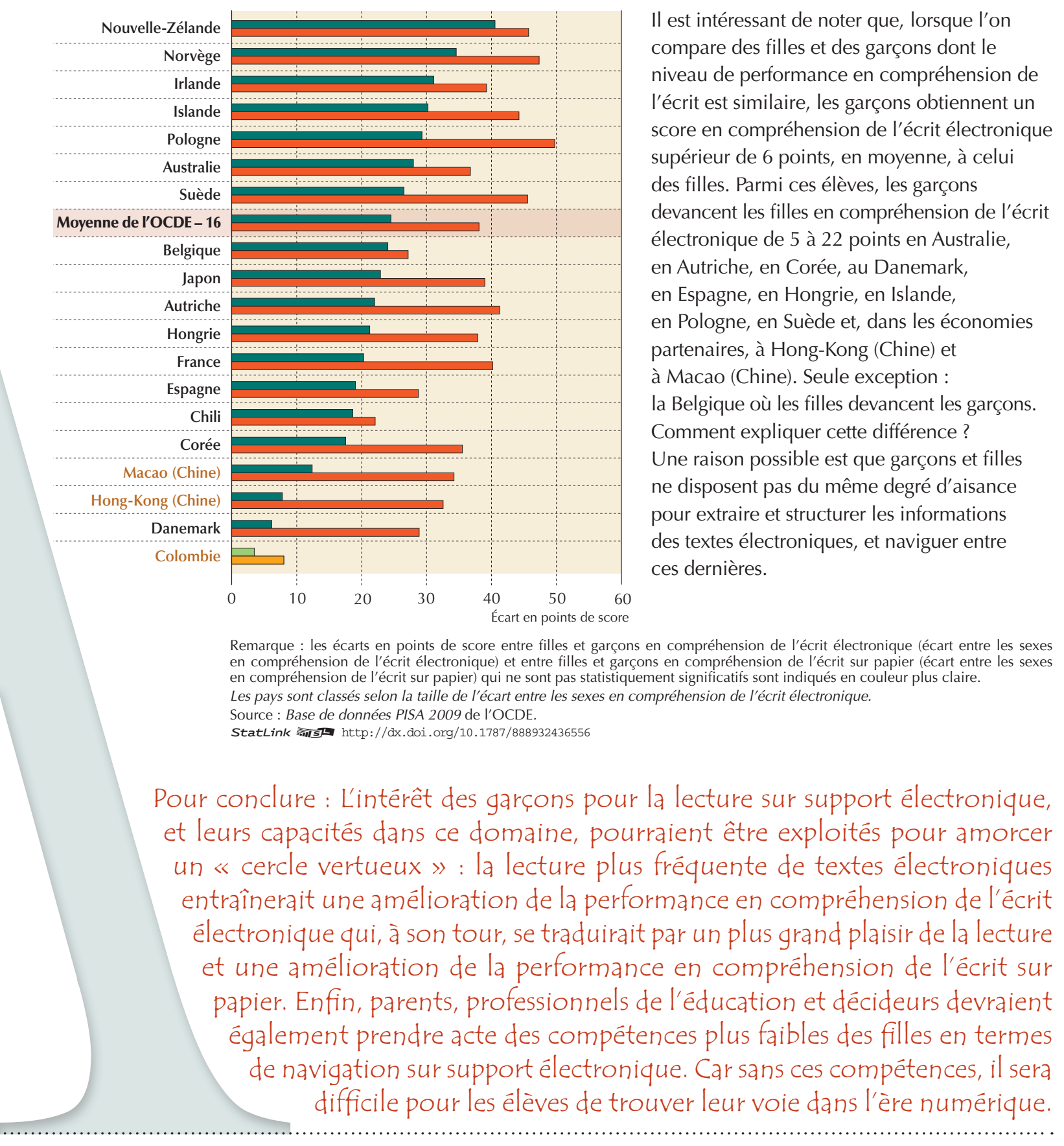

Pour tout complément d'information

Contacter Sophie Vayssettes (Sophie.Vayssettes@oecd.org)

Consulter Résultats du PISA 2009 : Élèves en ligne : Technologies numériques et performance (Volume VI)

\begin{tabular}{ll}
\hline voir & $\begin{array}{l}\text { Prochain numéro } \\
\text { www.pisa.oecd.org }\end{array}$ \\
www.oecd.org/pisa/infocus & Argent rime-t-il avec bonne performance \\
& dans l'enquête PISA? \\
\hline
\end{tabular}

